\title{
ALIGNMENT TRENDS OF VELOCITY GRADIENTS AND SUBGRID-SCALE FLUXES IN THE TURBULENT ATMOSPHERIC BOUNDARY LAYER
}

\author{
CHAD W. HIGGINS ${ }^{1,2, \star}$, MARC B. PARLANGE ${ }^{1,2}$ and C. MENEVEAU ${ }^{1,3}$ \\ ${ }^{1}$ Center for Environmental and Applied Fluid Mechanics, Johns Hopkins University, Baltimore, MD \\ 21218, U.S.A.; ${ }^{2}$ Department of Geography and Environmental Engineering, Johns Hopkins \\ University, Baltimore, MD, 21218, U.S.A., ${ }^{3}$ Department of Mechanical Engineering, Johns Hopkins \\ University, Baltimore, MD 21218, U.S.A.
}

(Received in final form 8 January 2003)

\begin{abstract}
Field experimental data in the atmospheric surface layer are analyzed using tools from statistical geometry. The data consist of velocity measurements from sonic anemometer arrays. In the context of large eddy simulations (LES), these arrays permit the spatial filtering needed to separate large from small scales. Time series of various quantities relevant to LES are evaluated from the data. Results show that the preferred filtered fluid deformation is axisymmetric extension and the preferred subgrid stress state is axisymmetric contraction. The filtered fluctuating vorticity shows preferred alignments with the mean vorticity, with the streamwise direction, and with the intermediate strain-rate eigenvector. The alignment between eigenvectors of the subgrid-scale stress and filtered strain rate is used to test eddy viscosity and mixed model formulations. In qualitative agreement with prior laboratory measurements at much lower Reynolds numbers, a bimodal distribution is observed, which can be reduced to good alignment with eddy viscosity closure using the mixed model.
\end{abstract}

Keywords: Atmospheric turbulence, Large eddy simulation, Statistical geometry, Subgrid-scale modelling.

\section{Introduction}

In large eddy simulation (LES) of turbulence, including the simulation of the atmospheric boundary layer (ABL), the subgrid-scale-stress tensor (SGS)

$$
\tau_{i j}=\widetilde{u_{i} u_{j}}-\tilde{u}_{i} \tilde{u}_{j}
$$

must be parameterized in terms of the resolved fields. A tilde $(\sim)$ represents spatial filtering at a scale $\Delta$ comparable with the numerical grid spacing used in the LES, and $u_{i}$ is the velocity vector field. The formulation of $\tau_{i j}$ reflects the multiple length scales that are inherent to turbulence (the above having filtered products and products of filtered velocity), and its three-dimensional properties (the SGS stress being a second rank tensor).

^ Corresponding author, E-mail: chad@jhu.edu 
Closure expressions are similarly needed for scalar turbulent transport:

$$
q_{i}=\widetilde{T u_{i}}-\tilde{T} \tilde{u}_{i},
$$

where the above expression is the SGS heat flux per unit heat capacity. Closure requires that the above quantities be expressed in terms of resolved scale variables such as the strain rate tensor,

$$
\tilde{S}_{i j}=\frac{1}{2}\left(\frac{\partial \tilde{u}_{i}}{\partial x_{j}}+\frac{\partial \tilde{u}_{j}}{\partial x_{i}}\right),
$$

the temperature gradient vector, or other Galilean-invariant properties of the resolved fields or domain geometry. Several recent articles on LES give a general overview of issues associated with SGS modelling and measurement interpretation (Piomelli, 1999; Meneveau and Katz, 2000; Porté-Agel et al., 2000a; Stevens and Lenschow, 2001).

Important features of SGS fluxes have recently been studied based on field experimental measurements in the ABL: Porté-Agel et al. (1998, 2000b, 2001a, b) and Tong et al. (1999). The emphasis of the analysis of Porté-Agel et al. was on statistical properties of so-called subgrid dissipation (i.e., the impact of the fluxes upon budgets of turbulent kinetic energy and scalar variance), as well as the effects of atmospheric stability and commonly observed coherent structures (onset of sweeps and ejections) upon relationships between resolved and subgrid variables.

The study of interactions among scales in turbulence has been enhanced by new measurement methods applied in detailed laboratory experiments. Specifically, the recent analysis carried out on holographic particle image velocimetry (HPIV) data by Tao et al. (2002) has provided remarkable insight into current, commonly used closure models and extends previous understanding of subgrid-scale physics obtained from low Reynolds number direct numerical simulation (DNS) studies. The three-dimensional velocity field measured by Tao et al. (2002) permits detailed studies of various model formulations. Briefly, their overall measurement domain covered a $57 \times 57 \times 45 \mathrm{~m}^{3}$ volume in the core of a turbulent square duct flow. They used a spatial filter size of $\Delta=3.3 \mathrm{~mm}$ with the Kolmogorov scale $\sim 0.1$ $\mathrm{mm}$. The Taylor-scale Reynolds number was $R_{\lambda} \approx 260$. Geometric features of SGS motions were studied by deducing the relative geometric alignment angles between the eigendirections of the SGS stress tensor and those predicted by various SGS models. Statistical geometric alignment trends were studied by measuring joint probability density functions of the three relevant alignment angles. They found a bimodal distribution of preferred alignment angles, and determined that the mixed model (a linear combination of a scalar and tensor eddy viscosity, see below) gave good predictions of the observed alignment trends. Moreover, they observed that axisymmetric extension was the preferred flow topology of the filtered velocity field. 
The results of Tao et al. (2002) motivate the study of alignment trends in other flow situations and on different spatial scales. In this paper we apply the analysis techniques used by Tao et al. (2002) to investigate alignment trends for turbulence in the atmospheric surface layer on scales about three orders of magnitude greater than those interrogated by the HPIV data (with filter scales $\Delta$ on the order of several metres as opposed to millimetres). We make use of the field data collected in Davis, California in the summer of 1999 (Porté-Agel et al., 2001a, b; Pahlow et al., 2001). The new analysis tools of Tao et al. (2002) are well suited for characterizing flow processes at the filtered scale (e.g., relationships between filtered temperature gradient and filtered strain rate), and in determining the alignments of the SGS stress and filtered strain rate with the mean flow directions. In the sections that follow, we provide a general overview of the data set of velocity and temperature, collected in the atmospheric surface layer using arrays of sonic anemometers. Then the analysis methodology is described. Observations of the flow properties, and $a$ priori tests of closure models are presented, and where possible compared to results obtained by Tao et al. (2002).

\section{Data Sets and Basic Characterization}

Two horizontal arrays of sonic anemometers were used in an experiment undertaken in the summer of 1999 in Davis, California to obtain spatial measurements of temperature (T) and velocity $\left(u_{i}\right)$. A detailed account of the data set is provided in Porté-Agel et al. (2001a, b) and Pahlow et al. (2001). The lower array contained seven sonic anemometers, and centered above it was an upper array containing five sonic anemometers. The entire setup was oriented in a field such that the angle of attack of the prevailing wind was nearly perpendicular to the line joining the instruments in each array. High temporal resolution $(20 \mathrm{~Hz})$ data were logged, and for each realization in time the three components of velocity and the temperature were measured. See Porté-Agel et al. (2001a, b) or Pahlow et al. (2001) for more details on the experiment, including photographs presented in Porté-Agel et al. (2001b) of the instrumental setup.

In this paper we consider the subset of data taken under unstable atmospheric stability. The friction velocity $u_{*}=\left(\left\langle u^{\prime} w^{\prime}\right\rangle^{2}+\left\langle v^{\prime} w^{\prime}\right\rangle^{2}\right)^{1 / 4}$ and the Obukhov length $L=-\langle T\rangle u_{*}^{3} / \kappa g\left\langle T^{\prime} w^{\prime}\right\rangle$ were used to classify the data into subsets according to the values of $z / L$ where $z$ is the average height of the sensors above the ground. Primes denote fluctuating quantities, $\langle$.$\rangle represents averaging over time, \kappa$ is von Kármán's constant $(\kappa=0.4)$ and $g$ is the acceleration of gravity. Data sets with $z / L$ within a range of -0.6 to -0.07 are classified as corresponding to convective conditions and were included in the data analyzed here. The average friction velocity for the data used was $u_{*}=0.27 \mathrm{~m} \mathrm{~s}^{-1}$. Other subsets of near-neutral conditions were also considered but some of the statistics obtained were not fully converged to allow unambiguous conclusions to be drawn; therefore, the results are not presented. This 


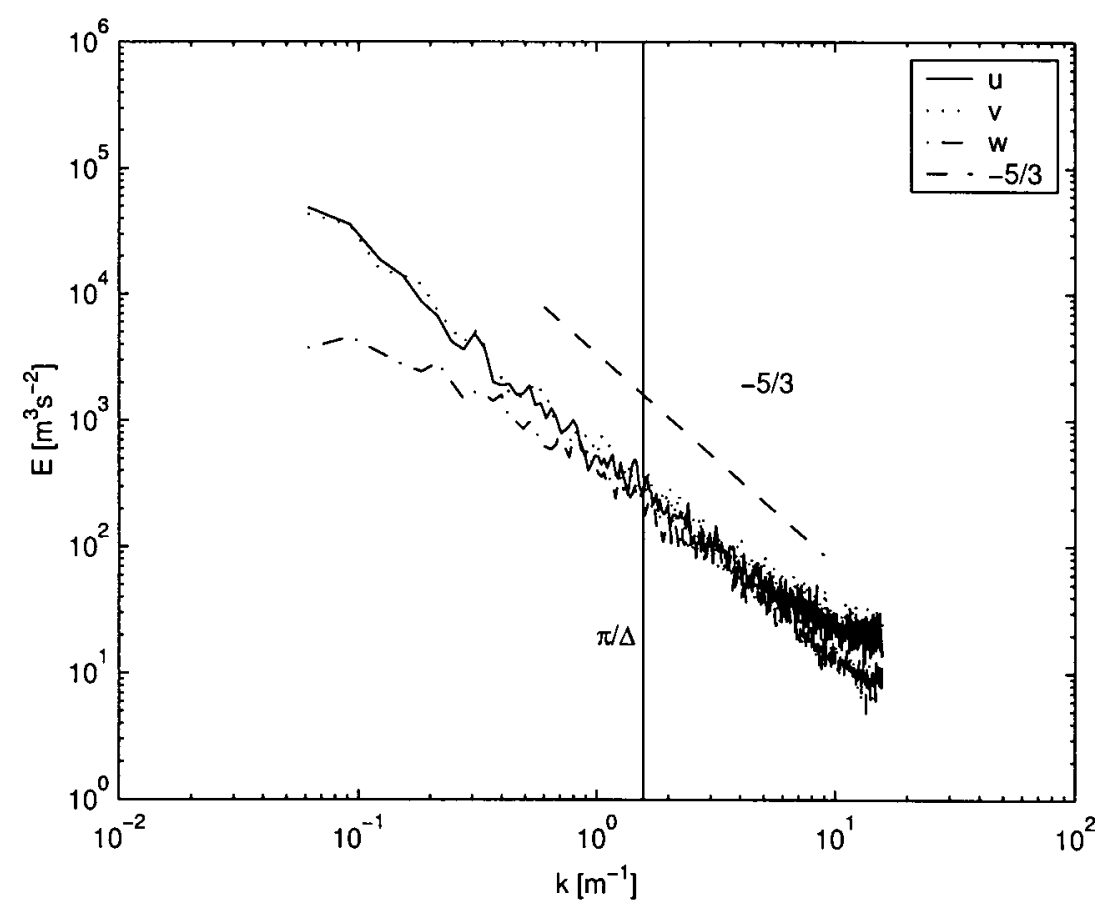

Figure 1. Spectra of a 30-min segment of data from the central sonic in the lower array. The height of the measurement from the ground is $3.8 \mathrm{~m}$. The spectra were calculated with linear detrending and windowing segments of 1024 samples with a Hanning window. As can be discerned from the spectra, the filter size lies within the inertial subrange, and we see a range of Kolmogorov scaling.

classification provided us with a total of about 900,000 data points, corresponding to about 32 segments of data (20 to 30 minutes long) covering a total of 11 hours of data (assembled from data taken over 10 days during June 1999).

For the direct calculation of the SGS fluxes (Equations (1) and (2)), the field measurements are filtered in the spanwise horizontal direction with a box filter, and in the streamwise direction with a Gaussian filter. Taylor's hypothesis is invoked to convert the temporal data record into a streamwise spatial record for streamwise filtering. The filter size used throughout the present work corresponds to five times the instrument spacing $\Delta \cong 2 \mathrm{~m}$, which lies in the inertial range of the turbulence spectra (see Figure 1). No filtering is performed in the vertical direction. For a comprehensive description of this approximate filtering technique, see Porté-Agel et al. (2001a) for applications to this same atmospheric data set, and Cerutti and Meneveau (2000) for applications to wind-tunnel laboratory data from arrays of hot-wire anemometers. 
The properties of the measured SGS stress are compared with tensors that arise in typical parameterizations. The classical base-line SGS model is the Smagorinsky model,

$$
\tau_{i j}^{s}=-2\left(c_{s} \Delta\right)^{2}|\tilde{S}| \tilde{S}_{i j},
$$

where $c_{s}$ is a dimensionless model parameter and $\tau_{i j}^{s}$ is the Smagorinsky parameterization for the deviatoric (traceless) part of the SGS stress tensor (the tensor in Equation (1) minus $\frac{1}{3} \tau_{k k} \delta_{i j}$ ). As reviewed in Meneveau and Katz (2000), on a local basis the model is known to exhibit a low correlation with the real stress, although in numerical simulations it performs better than these low correlations would indicate. This suggests that the three orthogonal eigenvectors of the resolved strain-rate tensor $\tilde{S}_{i j}$ provide a meaningful local coordinate frame for further analysis. Indeed, the analysis of Tao et al. (2002) uses this approach. For example, one may ask about the alignment of the real SGS stress eigenvectors with this local coordinate frame.

The nonlinear (or tensor eddy-viscosity) SGS parameterization (Leonard, 1974; Clark et al., 1979; Liu et al., 1994)

$$
\tau_{i j}^{n l}=C_{n l} \Delta^{2}\left(\frac{\partial \tilde{u}_{i}}{\partial x_{k}} \frac{\partial \tilde{u}_{j}}{\partial x_{k}}-\frac{1}{3} \frac{\partial \tilde{u}_{m}}{\partial x_{k}} \frac{\partial \tilde{u}_{m}}{\partial x_{k}} \delta_{i j}\right)
$$

yields significantly better results in correlation studies, and allows for the negative dissipation of turbulent kinetic energy. Although this latter feature is thought to be physically more realistic, negative dissipation can cause numerical errors to grow, leading to numerical instability. A third formulation, the so-called mixed model, is a linear combination of (3) and (4), and has been shown to perform well in a variety of flow situations when the dimensionless model coefficients are chosen to match the mean dissipation. (For a review of applications of the mixed model in LES see Meneveau and Katz, 2000). The models require evaluation of gradients of filtered velocity signals in all three directions. As in Porté-Agel et al. (2001a, b), vertical derivatives (in the $z$-direction) are calculated using finite difference approximations across the height separating the sensor arrays in the vertical direction. Transverse derivatives (in the $y$-direction) are calculated from data off the lower array by filtering using the first 5 and last 5 anemometers on the array and taking the difference. Streamwise derivatives are evaluated from temporal differences of filtered velocity. Taylor's hypothesis is invoked to convert temporal derivatives into stream-wise spatial derivatives. In order to provide approximately the same resolution in all directions, the distance across which finite differences are evaluated is $h=\Delta / 5$ in all three directions. Specifically, in the temporal (streamwise) direction, this distance is larger than the distance between the actual data points (separated by the finer data acquisition period corresponding to $20 \mathrm{~Hz}$ ). Where data are not available at exactly the points required (especially for filtering and differentiation in the $y$ direction), linear interpolation is used. The above treatment of the data allows for 
TABLE I

Basic Statistics of $\tilde{S}_{i j}$ and $\tau_{i j}$.

\begin{tabular}{llllllll}
\hline & $\tilde{S}_{11}$ & $\tilde{S}_{22}$ & $\tilde{S}_{33}$ & $\tilde{S}_{12}$ & $\tilde{S}_{13}$ & $\tilde{S}_{23}$ & {$\left[\mathrm{~s}^{-1}\right]$} \\
\hline Mean & 0.00 & 0.00 & 0.00 & 0.00 & 0.15 & 0.00 & \\
RMS & 0.11 & 0.22 & 0.18 & 0.14 & 0.19 & 0.18 & \\
\hline & $\tau_{11}$ & $\tau_{22}$ & $\tau_{33}$ & $\tau_{12}$ & $\tau_{13}$ & $\tau_{23}$ & {$\left[\mathrm{~m}^{2} \mathrm{~s}^{-2}\right]$} \\
\hline Mean & 0.16 & 0.14 & 0.079 & -0.0088 & -0.027 & 0.0019 & \\
RMS & 0.21 & 0.21 & 0.071 & 0.13 & 0.055 & 0.045 & \\
\hline & $\tau_{11}-\tau^{d}$ & $\tau_{22}-\tau^{d}$ & $\tau_{33}-\tau^{d}$ & {$\left[\mathrm{~m}^{2} \mathrm{~s}^{-2}\right]$} & & & \\
\hline Mean & 0.037 & 0.0095 & -0.0465 & & & & \\
RMS & 0.12 & 0.12 & 0.11 & & & &
\end{tabular}

the complete resolution of the SGS stress, SGS heat flux, filtered strain rate, filtered vorticity, and filtered temperature gradient. In this paper we focus our attention on features of the velocity field.

To reduce bias errors (constant offsets that could be different from one anemometer to the next) the temporal mean value from each anemometer is subtracted from each data segment. As a consequence, the mean vertical shear for the data is zero and is not included in most of the analysis. Thus, from here on, $\tilde{S}_{i j}$ represents the fluctuating part of the filtered strain-rate tensor (mean subtracted).

To show the effects of mean shear on the fluctuating results, some of the analysis of the present work will be performed including the mean shear. To include mean quantities in the presence of possible bias errors, the temporal mean is still subtracted from each sensor, but the mean velocity from each array (upper and lower) is added to the individual signals of sensors in each respective array. Thus, $\left\langle\tilde{S}_{13}\right\rangle$ is the only strain-rate element with non-zero mean $\left(\left\langle\tilde{S}_{13}\right\rangle \approx 0.15 \mathrm{~s}^{-1}\right.$ over the entire data set.

Table I presents mean and RMS values for the filtered strain rate and SGS stress elements. As discussed earlier, the only filtered strain rate element having non-zero mean is $\left\langle\tilde{S}_{13}\right\rangle$. The RMS values of the diagonal elements of $\tilde{S}_{i j}$ show anisotropy since $\tilde{S}_{11}$ has an RMS that is half the value of $\tilde{S}_{22}$, while the off diagonal terms of $\tilde{S}_{i j}$ have similar RMS values. The mean values of the SGS stress tensor are of the expected order of magnitude. Specifically, the order of magnitude of diagonal elements of the SGS stress tensor in the inertial range can be estimated as squared velocity increments over $\Delta$, i.e., a second-order structure function at displacement $r=\Delta$, which is $\sim \epsilon^{2 / 3} \Delta^{2 / 3}$. Using $\epsilon \sim u_{*}^{3} / z$ yields the diagonal elements of order $(\Delta / z)^{2 / 3} u_{*}^{2} \approx 0.11 \mathrm{~m}^{2} \mathrm{~s}^{-2}$ in the present data. RMS values of the SGS stress 
elements show that $\tau_{33}$ has a much smaller RMS than either $\tau_{11}$ or $\tau_{22}$, which is consistent with the usual anisotropic behaviour of the Reynolds stress, e.g., near the ground. For the analysis to follow, we subtract one third of the trace of the SGS stress tensor from the diagonal elements. The same procedure is applied for the strain-rate tensor. While in theory the trace of $\tilde{S}_{i j}$ should vanish due to incompressibility, the presence of noise, measurement, and differentiation errors, result in a trace that is not exactly zero. Hence, $\frac{1}{3} \tilde{S}_{k k} \delta_{i j}$ is subtracted from the diagonal elements of $\tilde{S}_{i j}$. Table I also presents the statistics of the diagonal elements of $\tau_{i j}$ after $\tau^{d}=\frac{1}{3} \tau_{k k} \delta_{i j}$ has been subtracted.

\section{Eigenvalue Structure and SGS Dissipation}

Before addressing the angles that characterize alignment properties of tensors in Section 4, we first describe a scalar parameter that characterizes the relative magnitude of the eigenvalues of a tensor. Suppose that $\alpha_{s}, \beta_{s}$, and $\gamma_{s}$ are the eigenvalues of the strain rate tensor, ordered so that $\alpha_{s}>\beta_{s}>\gamma_{s}$, and for incompressible flow $\alpha_{s}+\beta_{s}+\gamma_{s}=0$ (this also holds by construction for our field data after the trace has been subtracted). Hence, $\alpha_{s}>0$, and $\gamma_{s}<0$, while $\beta_{s}$ can have either sign. The relative magnitudes of the positive and negative eigenvalues, and their frequency of occurrence, have been the subject of various studies in turbulence (Ashurst et al., 1987; Tsinober et al., 1992; Lund and Rogers, 1994). In particular, Lund and Rogers (1994) proposed a local non-dimensional parameter called the strain state parameter, $s^{*}$

$$
s^{*}=\frac{-3 \sqrt{6} \alpha_{s} \beta_{s} \gamma_{s}}{\left(\alpha_{s}^{2}+\beta_{s}^{2}+\gamma_{s}^{2}\right)^{3 / 2}} .
$$

The strain state parameter is bounded between -1 and 1 , and is useful since it indicates the type of deformation occurring at the location of the measured filtered strain rate. For example $s^{*}=1$ corresponds to axisymmetric extension (i.e., $\alpha_{s}=$ $\left.\beta_{s}>0, \gamma_{s}<0\right) ; s^{*}=0$ (i.e., $\beta_{s}=0$ ) corresponds to plane shear, and $s^{*}=-1$ (i.e., $\alpha_{s}>0, \beta_{s}=\gamma_{s}<0$ ) corresponds to axisymmetric contraction. Moreover, Lund and Rogers (1994) have shown that for random data the probability density function (PDF) of $s^{*}$ is an unbiased uniform distribution between -1 and 1 .

The strain state parameter $s^{*}$ is evaluated from the data at every data point, and the PDF of $s^{*}$ is constructed. The measured PDF is shown in Figure 2. The increased value around $s^{*}=1$ shows that the most likely structure of the filtered fluctuating strain rate in atmospheric surface-layer turbulence is axisymmetric extension. The same conclusion was reached for unfiltered turbulence at lower Reynolds numbers and smaller scales by Lund and Rogers (1994) (from DNS data), by Tsinober et al. (1992) (from multi component hot wire data), and by Tao et al. (2002) (for filtered data from three-dimensional (3-D) holographic particle-image velocimetry data in a square duct). 


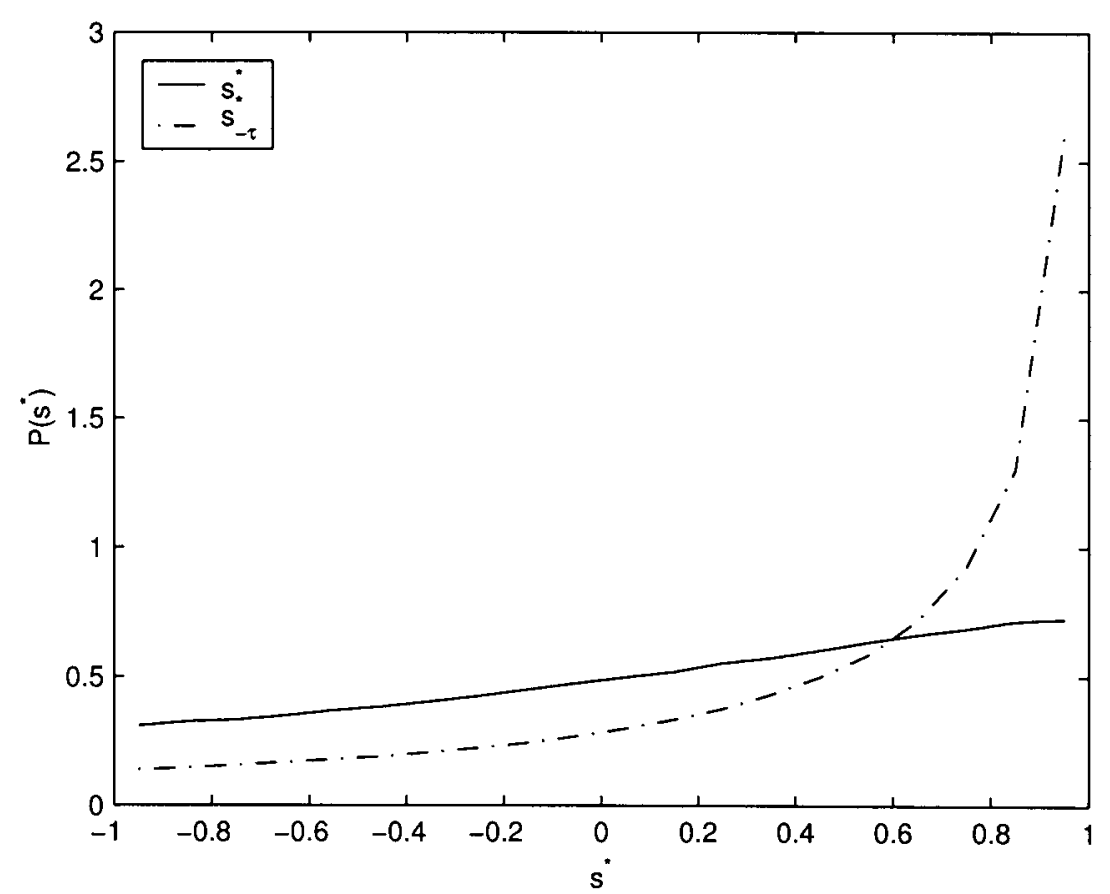

Figure 2. Probability density distribution of strain-rate state $s^{*}$ (solid line) and negative SGS stress tensor state (dashed line) obtained from filtered field experimental data in the atmospheric surface layer. The filter scale is $\Delta=2 \mathrm{~m}$. These probability density functions show that local axisymmetric extension is the most likely state of the strain-rate tensor, and local axisymmetric contraction is the most likely state of the SGS stress.

Another parameter, the SGS stress state parameter, $s_{-\tau}^{*}$, was used by Tao et al. (2002) to perform a similar characterization of the relative eigenvalues of the deviatoric part of the SGS stress tensor. The same formulation (Equation (5)) is used, but applied to the eigenvalues of the negative SGS stress tensor: $\alpha_{-\tau}, \beta_{-\tau}$, and $\gamma_{-\tau}$. Negative stress is chosen in view of the minus sign in Equation (3) associated with the classical eddy-viscosity closure.

The measured PDF of $s_{-\tau}^{*}$ (dot-dashed line in Figure 2) shows that the most likely state of the negative SGS stress is also axisymmetric extension. However this is interpreted as axisymmetric contraction for the positive SGS stress tensor as $s_{-\tau}^{*}=-s_{\tau}^{*}$, i.e., the SGS motions cause preferentially two inward forces of equal magnitude, with one stretching direction. Also, the peak in the PDF is more pronounced than the peak for the strain-rate tensor.

The SGS dissipation of energy from large to small scales defined as,

$$
\Pi=-\tau_{i j} \tilde{S}_{i j},
$$

is a critical issue in LES modelling (Meneveau and Katz, 2000). A probability density function of the local SGS dissipation (Figure 3) shows negative values (not 


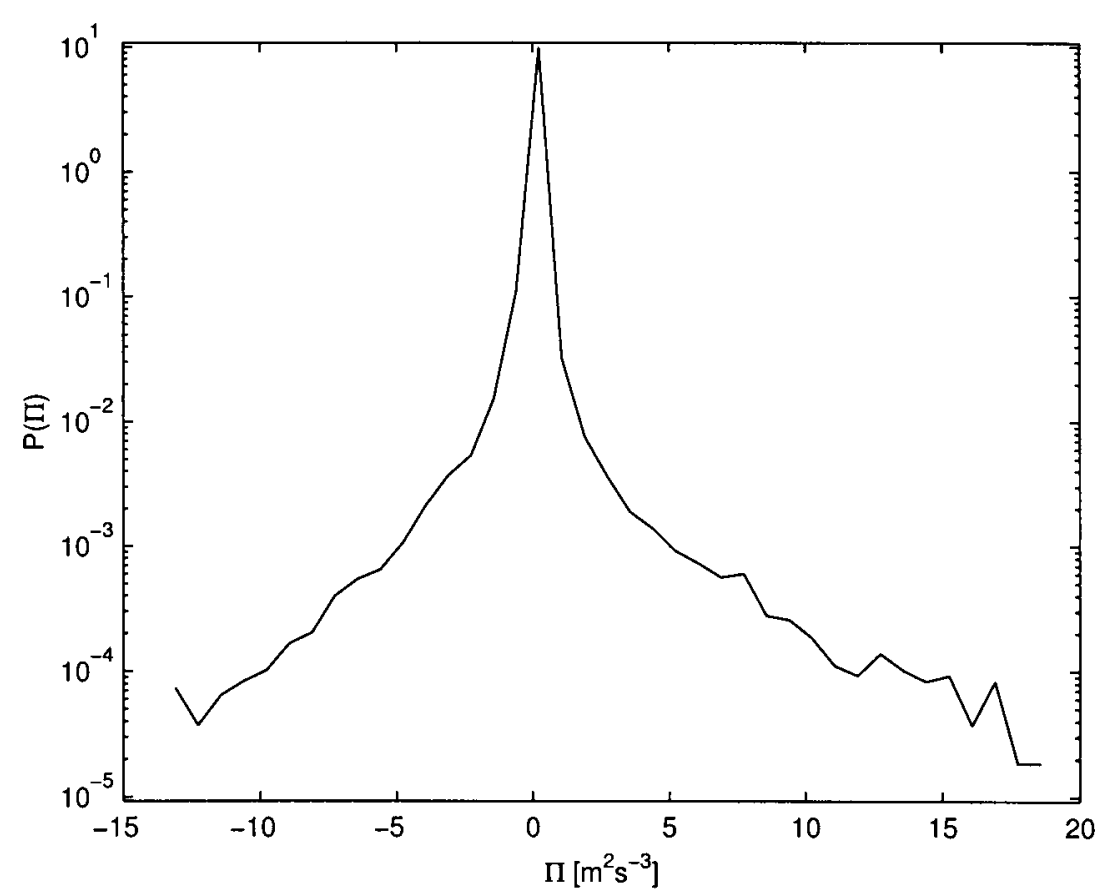

Figure 3. Probability density distribution of local SGS energy dissipation $\left(\Pi=-\tau_{i j} \tilde{S}_{i j}\right)$ measured from an array of sonic anemometers in the atmospheric surface layer under unstable conditions. Filter scale is $\Delta=2 \mathrm{~m}$. There is a considerable probability of energy backscatter, but the mean dissipation is positive, thus transferring energy from large to small scales.

reproducible by the Smagorinsky model) indicating localized backscatter of energy from small scales to large scales. The mean dissipation is positive, and therefore most of the kinetic energy is transferred from the resolved to subgrid-scale motions. Mean dissipation is often used to calibrate (Lilly, 1967), or to evaluate empirically (Porté-Agel et al., 2001a, b) the coefficients $c_{s}$, and $C_{n l}$ in Equations (3) and (4). Clearly the alignment between the eigenvectors of the SGS stress tensor and the strain-rate tensor will determine the magnitude of the tensors' inner product (Equation (6)). A subsequent publication will investigate the relationships between the eigenvalues and eigenvector structure of the strain rate, and the SGS stress on dissipation.

\section{Statistical Geometry Analysis Tools}

Closure for vector quantities, such as the equality of the SGS heat flux and its parameterizations, implies that both the direction and magnitude must match, i.e., perfect angular alignment and equality of magnitudes. In this section, we focus on purely geometrical alignment trends. These do not depend upon the model coefficients $c_{s}$ and $C_{n l}$ (except for the mixed model where the results depend upon the 


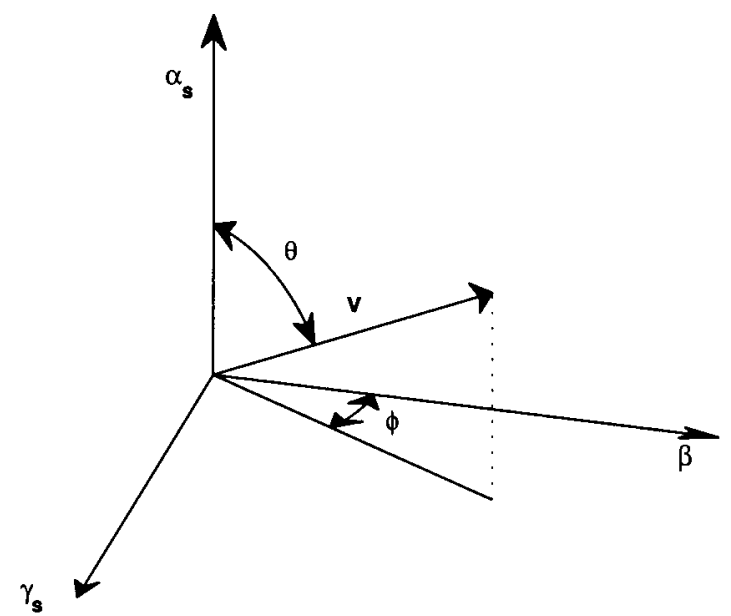

Figure 4. Definition of two angles to fully characterize relative alignment between a vector, $\mathbf{v}$, and an orthogonal (symmetric) tensor whose eigenvectors are $\boldsymbol{\alpha}, \boldsymbol{\beta}$, and $\boldsymbol{\gamma}$. These angle definitions are analogous to spherical coordinates and are consistent with definitions used in previous studies.

ratio of the coefficients). Here we present the analysis quantifying the orientation of a vector relative to a symmetric tensor, and its further extension to fix the relative alignment of two symmetric tensors. The relative orientation between two vectors in three-dimensional space is described by a single angle. To provide unbiased results, the PDF of the cosine of this angle is measured. For instance, for the vorticity $\boldsymbol{\omega}$, and the most extensive strain-rate eigenvector $\boldsymbol{\alpha}_{\tilde{S}}$, one measures

$$
\cos (\theta)=\frac{\left|\omega \cdot \alpha_{\tilde{S}}\right|}{|\omega|\left|\alpha_{\tilde{S}}\right|}
$$

and its PDF. For random vectors, such a PDF is uniform.

To characterize the alignments between a vector (e.g., vorticity or temperature gradient) and a tensor (e.g., strain rate or SGS stress), or between two tensors (e.g., strain rate and SGS stress) requires the specification of two or three angles, respectively.

The filtered strain rate and the SGS stress tensors are (by construction) symmetric and traceless, thus the eigenvectors of the tensors are both real and orthogonal (i.e., they form a basis of three-dimensional space). The directionality of any given vector relative to the eigenvector basis of the symmetric strain rate tensor can be specified by two angles (defined in Figure 4), as is common practice in spherical coordinates. The statistics of alignment trends between vectors and the strain-rate tensor basis are quantified using joint probability density functions of these two angles. This joint PDF is plotted on the unit sphere in order to emphasize the relative alignment, in physical space, of the two quantities.

Characterization of the full tensor-tensor alignment (e.g., SGS stress and strainrate tensor) is more complicated, and as will be shown below, requires the 


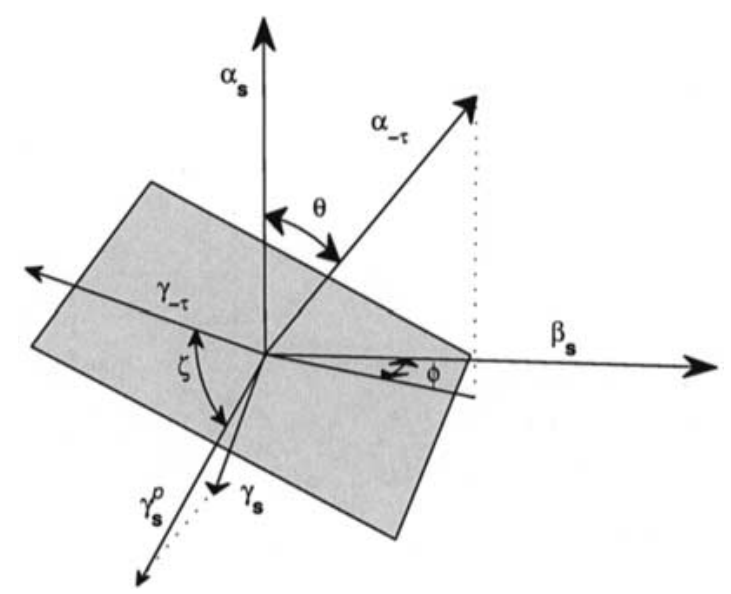

Figure 5. Definition of three angles to fully characterize relative alignment between two orthogonal (symmetric) tensors. The angles $\theta$ and $\phi$ are elevation and azimuth angles of the vector $\boldsymbol{\alpha}_{-\tau} \boldsymbol{\tau}$ relative to the filtered strain rate eigendirections. The angle $\zeta$ is the angle between $\gamma_{\tilde{S}}^{p}$, the projection of $\boldsymbol{\gamma}_{\tilde{\boldsymbol{S}}}$ onto the $\boldsymbol{\alpha}_{-\boldsymbol{\tau}} \boldsymbol{-} \boldsymbol{\beta}_{-\boldsymbol{\tau}}$ plane, and $\boldsymbol{\gamma}_{-\boldsymbol{\tau}}$. Joint probability density functions of $\cos (\theta), \phi$, and $\zeta$ yield unbiased results for randomly orientated tensors, and this angle convention allows for direct comparison with previous studies.

specification of three angles (see Figure 5). We begin by choosing an eigenvector of the SGS stress (e.g., $\boldsymbol{\alpha}_{-\tau}$ ) and describe its orientation in the strain rate eigensystem $\boldsymbol{\alpha}_{\tilde{S}}, \boldsymbol{\beta}_{\tilde{S}}$, and $\boldsymbol{\gamma}_{\tilde{S}}$. Thus, the extensive eigendirection of the SGS stress, $\boldsymbol{\alpha}_{-\tau}$, is described in spherical coordinates relative to the strain rate eigendirections by specifying two angles

$$
\begin{aligned}
& \theta=\cos ^{-1} \frac{\left|\boldsymbol{\alpha}_{\tilde{S}} \cdot \boldsymbol{\alpha}_{-\tau}\right|}{\left|\boldsymbol{\alpha}_{\tilde{S}}\right|\left|\boldsymbol{\alpha}_{-\tau}\right|}, \\
& \phi=\cos ^{-1} \frac{\mid \boldsymbol{\beta}_{\tilde{S}} \cdot\left(\boldsymbol{\alpha}_{-\tau}-\left(\boldsymbol{\alpha}_{-\tau} \cdot \boldsymbol{\alpha}_{\tilde{S}}\right) \boldsymbol{\alpha}_{\tilde{S}} \mid\right.}{\left|\left(\boldsymbol{\alpha}_{-\tau}-\left(\boldsymbol{\alpha}_{\tilde{S}} \cdot \boldsymbol{\alpha}_{-\tau}\right) \boldsymbol{\alpha}_{\tilde{S}}\right)\right|\left|\boldsymbol{\beta}_{\tilde{S}}\right|} .
\end{aligned}
$$

Still, the orientation of the SGS stress relative to the filtered strain rate has not yet been fixed completely since the contractive, $\boldsymbol{\gamma}_{-\tau}$, and intermediate, $\boldsymbol{\beta}_{-\tau}$, eigendirections are free to rotate in a plane perpendicular to $\alpha_{-\tau}$ (see Figure 5). In order to fix the orientation of (say) $\gamma_{-\tau}$ on this plane perpendicular to $\alpha_{-\tau}$, a reference direction is required. A possibility would be the contracting strain rate direction $\boldsymbol{\gamma}_{\tilde{S}}$, although there is no guarantee that $\boldsymbol{\gamma}_{\tilde{S}}$ is on the $\boldsymbol{\gamma}_{-\tau}, \boldsymbol{\beta}_{-\tau}$ plane. To provide a unique reference direction on the $\boldsymbol{\gamma}_{-\tau}, \boldsymbol{\beta}_{-\tau}$ plane, the contracting eigendirection of the filtered strain rate, $\boldsymbol{\gamma}_{\tilde{S}}$, is projected into the plane perpendicular to the fixed SGS stress eigendirection $\boldsymbol{\alpha}_{-\tau}$. This projection now lies in the plane spanned by two eigendirections of the SGS stress $\boldsymbol{\gamma}_{-\tau}$ and $\boldsymbol{\beta}_{-\tau}$, and its angle,

$$
\zeta=\cos ^{-1} \frac{\left|\boldsymbol{\gamma}_{-\tau} \cdot\left(\boldsymbol{\gamma}_{\tilde{S}}-\left(\boldsymbol{\gamma}_{\tilde{S}} \cdot \boldsymbol{\alpha}_{-\tau}\right) \boldsymbol{\alpha}_{-\tau}\right)\right|}{\left|\boldsymbol{\gamma}_{-\tau}\right| \mid\left(\boldsymbol{\gamma}_{\tilde{S}}-\left(\boldsymbol{\gamma}_{\tilde{S}} \cdot \boldsymbol{\alpha}_{-\tau}\right) \boldsymbol{\alpha}_{-\tau} \mid\right.}
$$


relative to the contracting direction of the SGS stress, $\boldsymbol{\alpha}_{-\tau}$, now completes the description of the relative alignment of the two tensors. Algebraically, this can be interpreted as solving for a rotation matrix that transforms one set of eigendirections into the other. The results of this analysis are presented as the 3-D joint PDF of $\cos (\theta), \phi$, and $\zeta$ defined as $P(\cos \theta, \phi, \zeta)$. These variables produce unbiased results, i.e., $P(\cos \theta, \phi, \zeta)$ is uniform when applied to randomly oriented data. Additional details of the analysis technique are presented in Tao et al. (2002). Presently, 3-D joint PDFs are calculated from the data by dividing the variable ranges into 10 bins, and accumulating statistics over all points of the signals.

\section{Results}

\subsection{Filtered SCALE OBSERVATIONS}

The first, and most intuitive, application of the methods described in Section 4 is in the statistical description of the orientation of the filtered fluctuating vorticity vector. We present the alignment of filtered vorticity with flow coordinates (i.e., a fixed coordinate system aligned with the mean flow), the alignment of filtered vorticity and the filtered strain rate eigendirections (i.e., a varying local coordinate system depending on the instantaneous alignments of $\tilde{S}_{i j}$ ), and the alignment of filtered strain rate eigendirections with the flow coordinate system.

The joint PDF characterizing the alignment of filtered fluctuating vorticity with the mean-flow defined coordinate system exhibits a bimodal behaviour, Figure 6a. The primary alignment trend observed is for the fluctuating filtered vorticity to be aligned with the cross-stream $y$-direction. In any boundary layer with the mean velocity aligned in the $x$-direction and $z$ being the wall normal direction, the mean vorticity is aligned with the $y$-axis. Therefore, the primary alignment trend in Figure 6a shows that the most likely orientation of the fluctuating turbulent filtered vorticity vector is aligned with the mean vorticity (associated with boundary-layer behaviour). The secondary local peak corresponds to stream-wise vorticity pointing in the $x$-direction. It is well known that turbulent boundary layers have distinct stream-wise vortices (Cantwell, 1981; Bernal and Roshko, 1986; Lasheras and Choi, 1988; Bell and Metha, 1992; Lin, 1999; Finnigan, 2000) and the results here show that such structures are present in the fluctuating filtered vorticity field.

Investigations concerning the alignment of vorticity and the eigenvectors of the filtered strain rate have already received considerable attention. Ashurst et al. (1987) observed that vorticity was aligned with the intermediate eigendirection, $\left(\boldsymbol{\beta}_{\tilde{S}}\right)$, of the strain-rate tensor in DNS data. Tsinober et al. (1992) found similar results from hot-wire measurements. Tao et al. (2002) observed the same result based on their filtered holographic particle image velocimetry (HPIV) measurements. We repeat the analysis of Tao et al. here using the atmospheric data obtained from arrays of sonic anemometers. Figure $6 \mathrm{~b}$ shows a very strong peak in the measured 
joint PDF of angles between filtered fluctuating vorticity and filtered fluctuating strain-rate basis. The filtered fluctuating vorticity is clearly preferentially aligned with the intermediate direction of the filtered fluctuating strain rate.

In Figure 7 the alignment of the filtered strain rate with the mean flow coordinate system is plotted. Like the filtered fluctuating vorticity, the filtered strain rate exhibits a bimodal alignment trend. The primary peak (a) is consistent with a boundary-layer behaviour (but again note that the mean strain rate has been subtracted from the signals and is thus not included in the present analysis). In this alignment trend, the intermediate eigendirection, $\boldsymbol{\beta}_{\tilde{S}}$, is aligned with the crossstream direction while the extending direction, $\alpha_{\tilde{S}}$, is $45^{\circ}$ from the vertical in the downstream direction. At the same time the most contracting eigendirection, $\boldsymbol{\gamma}_{\tilde{S}}$, is aligned $45^{\circ}$ from the vertical. This is the alignment for an ideal boundary layer in which only a vertical gradient of velocity exists. This behaviour is consistent with the mean strain in which the only non-zero strain element is $\left\langle\tilde{S}_{13}\right\rangle$. Thus, for the primary peak in alignment, the filtered fluctuating strain is aligned with the mean straining directions, and the mean straining directions are characteristic of an ideal boundary-layer flow. In Figures $6 \mathrm{a}$ and $\mathrm{b}$ we showed that the fluctuating filtered vorticity is aligned with $\boldsymbol{\beta}_{\tilde{S}}$ and the cross-stream horizontal direction (the primary alignment of vorticity with flow defined coordinates). The observed alignment of $\boldsymbol{\beta}_{\tilde{S}}$ with the cross-stream direction (again one of two possible alignment configurations of filtered strain rate and Cartesian coordinates) is consistent with the previous observations of filtered fluctuating vorticity. A composite picture of the primary alignments of fluctuating vorticity, fluctuating strain, and the flow defined coordinate system is presented in Figure 8a.

The secondary alignment trend (b) in Figure 7 is interpreted as the intermediate eigendirection, $\boldsymbol{\beta}_{\tilde{S}}$, aligned with the stream-wise direction while the extensive and contracting directions are tilted $45^{\circ}$ from the vertical as they lie in the $y$ - $z$ plane. In Figures $6 a$ and $b$, the secondary alignment of filtered fluctuating vorticity with flow defined coordinates was that the fluctuating filtered vorticity be aligned with $\boldsymbol{\beta}_{\tilde{S}}$ and the streamwise direction. The observed alignment of $\boldsymbol{\beta}_{\tilde{S}}$ with the streamwise direction in this secondary configuration is consistent with the previous observations of filtered fluctuating vorticity. Combining information deduced from Figures $6 \mathrm{a}, 6 \mathrm{~b}$, and 7 shows that in the secondary alignment of filtered quantities: (1) the filtered fluctuating vorticity, (2) the intermediate eigendirection of the fluctuating filtered strain $\boldsymbol{\beta}_{\tilde{S}}$, and (3) the streamwise direction are colinear. A composite picture of the secondary alignments of fluctuating vorticity, fluctuating strain, and the flow defined coordinate system is presented in Figure 8b.

\subsection{Alignments BetweEn FILTERED SCALE AND SUbGRID-SCALE QUANTITIES}

In this section, we describe the observed alignments between filtered and SGS quantities. Here we are concerned with the alignment of filtered vector quantities, 

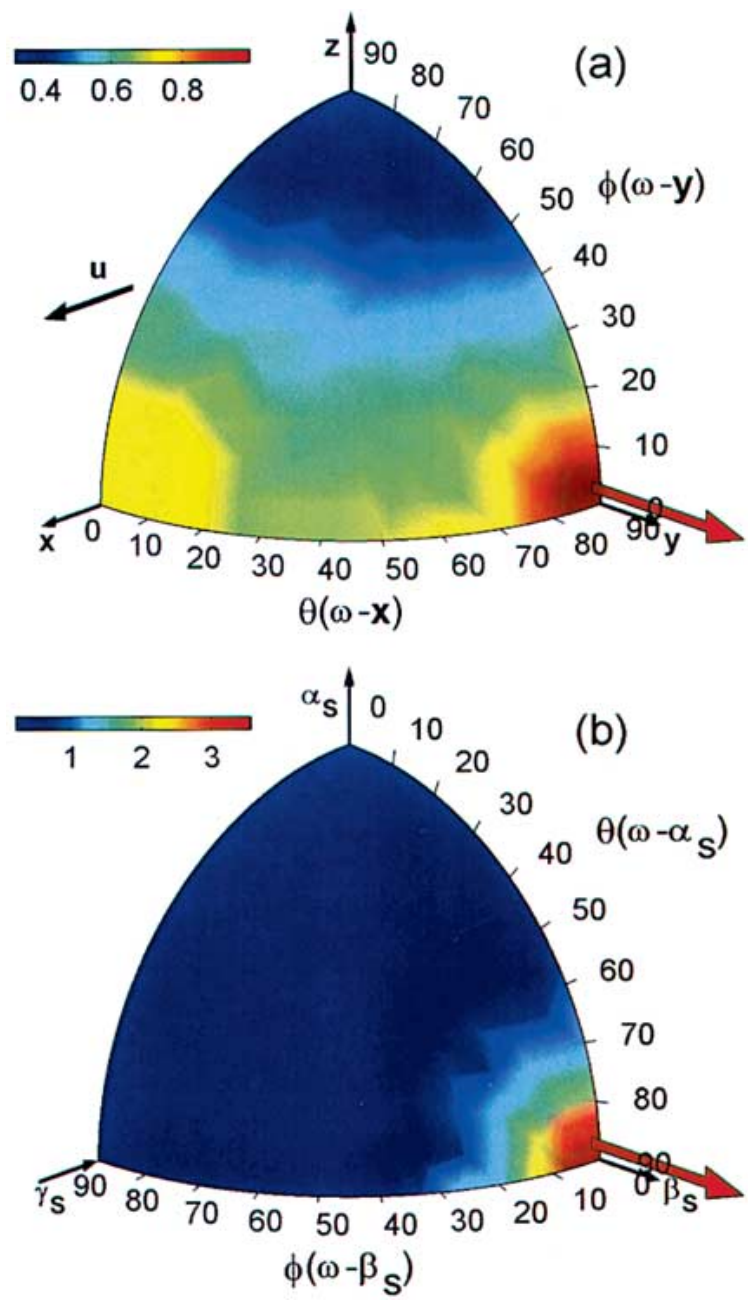

Figure 6. (a) Joint probability density function of two angles describing the orientation of filtered fluctuating vorticity vector in the mean flow frame of reference. Filter scale is $\Delta=2 \mathrm{~m}$. The mean flow streamwise direction is $x$, and $z$ is the vertical axis. The bimodal distribution shows two likely orientations of filtered fluctuating vorticity. In the primary configuration, vorticity is aligned with the cross-stream horizontal direction, while in the secondary configuration, vorticity is aligned with the streamwise direction. (b) Joint PDF of two angles describing the orientation of filtered fluctuating vorticity vector in local strain-rate tensor eigensystem. The $\boldsymbol{\beta} \tilde{\boldsymbol{s}}$-axis corresponds to the strain-rate eigendirection associated with the intermediate eigenvalue of the filtered fluctuating strain-rate tensor. This figure shows the strong alignment of filtered vorticity with the intermediate eigendirection of the fluctuating filtered strain rate, $\boldsymbol{\beta}_{\tilde{\boldsymbol{S}}}$. 


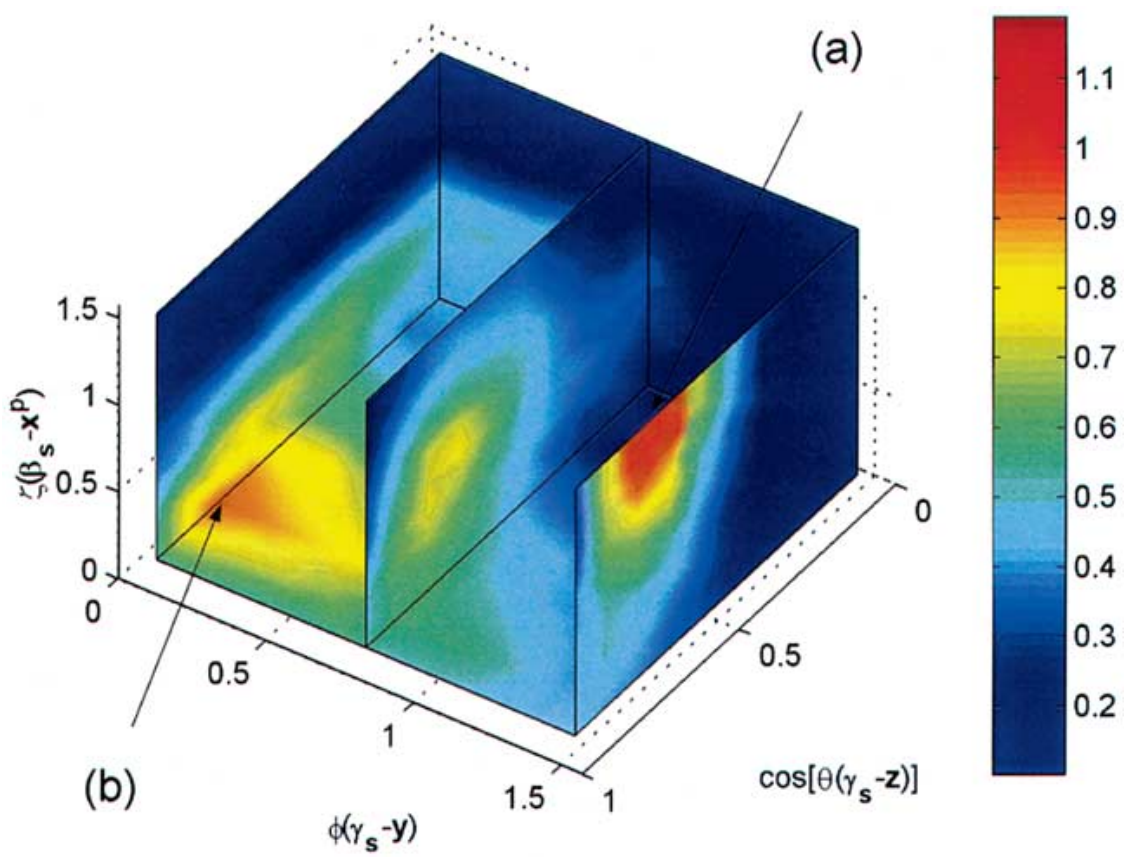

Figure 7. Joint probability density function of three angles describing the orientation of filtered fluctuating strain-rate tensor eigensystem in mean flow frame of reference. Filter scale is $\Delta=2 \mathrm{~m}$. The mean flow streamwise direction is $x, z$ is the vertical axis, and $y$ is the transverse horizontal direction. Here, the planes represent slices through the three-dimensional function at locations where the modes of probability lie. The two modes present in this alignment are consistent with the vorticity behaviour (see text).
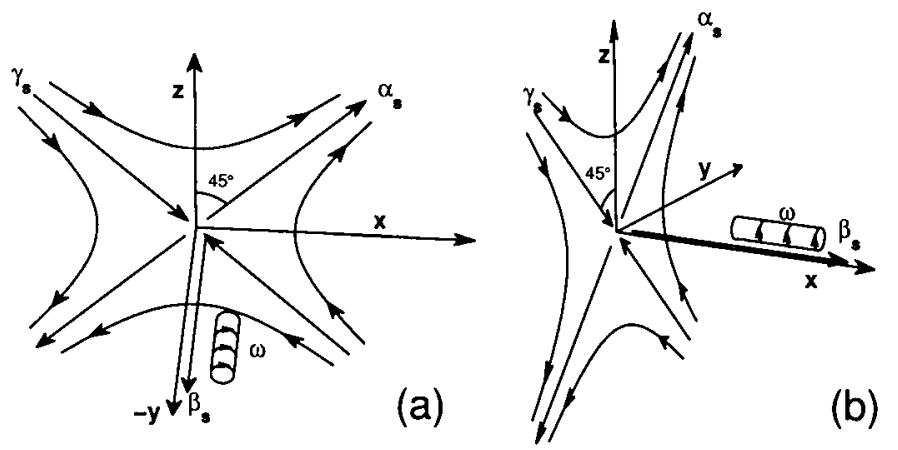

Figure 8. Sketch of two most likely alignments of filtered fluctuating vorticity, filtered fluctuating strain rate eigendirections, and the mean flow coordinate system. Composite alignments are deduced from individual alignments presented in Figures $6 \mathrm{a}, 6 \mathrm{~b}$ and 7 , and show the two most likely orientations of all the filtered quantities analyzed here. Sketch (a) shows the primary alignment trend, and (b) shows the secondary alignment trend. 
such as the filtered vorticity, as described within a local orthogonal coordinate system given by the eigenvectors of the SGS stress tensor. The joint PDF of angles of filtered vorticity relative to eigenvectors of the (negative) SGS stress tensor is shown in Figure 9a. A bimodal distribution is apparent. The strongest PDF peak shows the preferential alignment of filtered fluctuating vorticity with the intermediate eigendirection of the negative SGS stress, $\boldsymbol{\beta}_{-\tau}$. Tao et al. (2002) also observed this alignment trend in their laboratory data. The secondary peak shows the fluctuating filtered vorticity is aligned with the most extensive direction of the negative SGS stress, $\boldsymbol{\alpha}_{-\tau}$, which is the most contracting direction of the positive SGS stress (this secondary peak was not apparent in the data of Tao et al. (2002)). Recall from Figure 2 that the stress-state parameter had a most likely value of $1\left(s_{-\tau}^{*}=1\right)$. This indicates that the most likely state of negative SGS stress is axisymmetric extension, meaning that both $\boldsymbol{\alpha}_{-\boldsymbol{\tau}}$ and $\boldsymbol{\beta}_{-\boldsymbol{\tau}}$ are contracting at the same magnitude most of the time. Therefore, in the most likely sense, the fluctuating filtered vorticity is preferentially aligned with a contracting direction of the SGS stress. This can also be understood as the filtered vorticity is preferentially perpendicular to the extensive SGS stress eigendirection.

In Figure $9 b$ the results characterizing the alignment of the SGS stress with the mean flow directions are presented. Clearly there are preferred orientations, showing that in this flow the mean shear retains significant influence in determining small-scale statistics such as SGS stress-tensor orientations. The results indicate that the intermediate eigendirection of the SGS stress, $\boldsymbol{\beta}_{-\tau}$, is aligned with the cross-stream horizontal direction. The most extensive SGS stress eigendirection, $\boldsymbol{\gamma}_{-\tau}$, and the most contracting SGS eigendirection, $\boldsymbol{\alpha}_{-\tau}$, are confined to the $x-z$ plane. $\boldsymbol{\gamma}_{-\tau}$ is inclined at about 75 degrees from the vertical direction, 15 degrees from the horizontal streamwise direction, and $\boldsymbol{\alpha}_{-\tau}$ is aligned 15 degrees from the horizontal in the upstream direction. If we again consider the alignments of vorticity, SGS stress, and mean flow directions we see that the filtered fluctuating vorticity, the intermediate eigendirection of the SGS stress, and the horizontal cross-stream direction are all most likely co-linear.

Given the prominence of the Smagorinsky model in LES, the measurement of alignment trends between the negative SGS stress tensor and the filtered strain rate is of particular importance. The three-dimensional tensor-tensor alignment of fluctuating filtered strain rate, $\tilde{S}_{i j}$, and negative SGS stress, $-\tau_{i j}$, is presented in Figure 10. It is apparent that, in terms of preferred alignment, there are two relative alignments displaying elevated levels of probability. The first peak, denoted by (a), gives the strongest trend, is interpreted as $\alpha_{-\tau}$, is preferentially aligned at about $28^{\circ}$ from $\boldsymbol{\alpha}_{\tilde{S}}\left(\cos \left[\theta\left(\boldsymbol{\alpha}_{-\tau}-\boldsymbol{\alpha}_{\tilde{S}}\right)\right] \approx 0.88\right)$ while simultaneously having a projection onto the $\boldsymbol{\gamma}_{\tilde{S}}-\boldsymbol{\beta}_{\tilde{S}}$ plane that is aligned with $\boldsymbol{\gamma}_{\tilde{S}}\left(\phi\left[\boldsymbol{\alpha}_{-\tau}-\boldsymbol{\beta}_{\tilde{S}}\right] \approx 90^{\circ}\right)$ and the angle $\zeta\left[\gamma_{-\tau} \gamma_{\tilde{S}^{p}}\right] \approx 0^{\circ}$. The second, slightly weaker peak (b) is given by the trio of angles $\cos \left[\theta\left(\boldsymbol{\alpha}_{-\tau}-\boldsymbol{\alpha}_{\tilde{S}}\right)\right] \approx 0, \phi\left[\boldsymbol{\alpha}_{-\tau}-\boldsymbol{\beta}_{\tilde{S}}\right] \approx 0^{\circ}$, and $\zeta\left[\boldsymbol{\gamma}_{-\tau}-\boldsymbol{\gamma}_{\tilde{\boldsymbol{S}}}^{p}\right] \approx 30^{\circ}$. Interpretive sketches of the alignment trend observed in each respective case are presented in Figure 10. The difference between configurations (a) and (b) is that $\boldsymbol{\alpha}_{-\tau}$ and $\boldsymbol{\beta}_{-\boldsymbol{\tau}}$ 

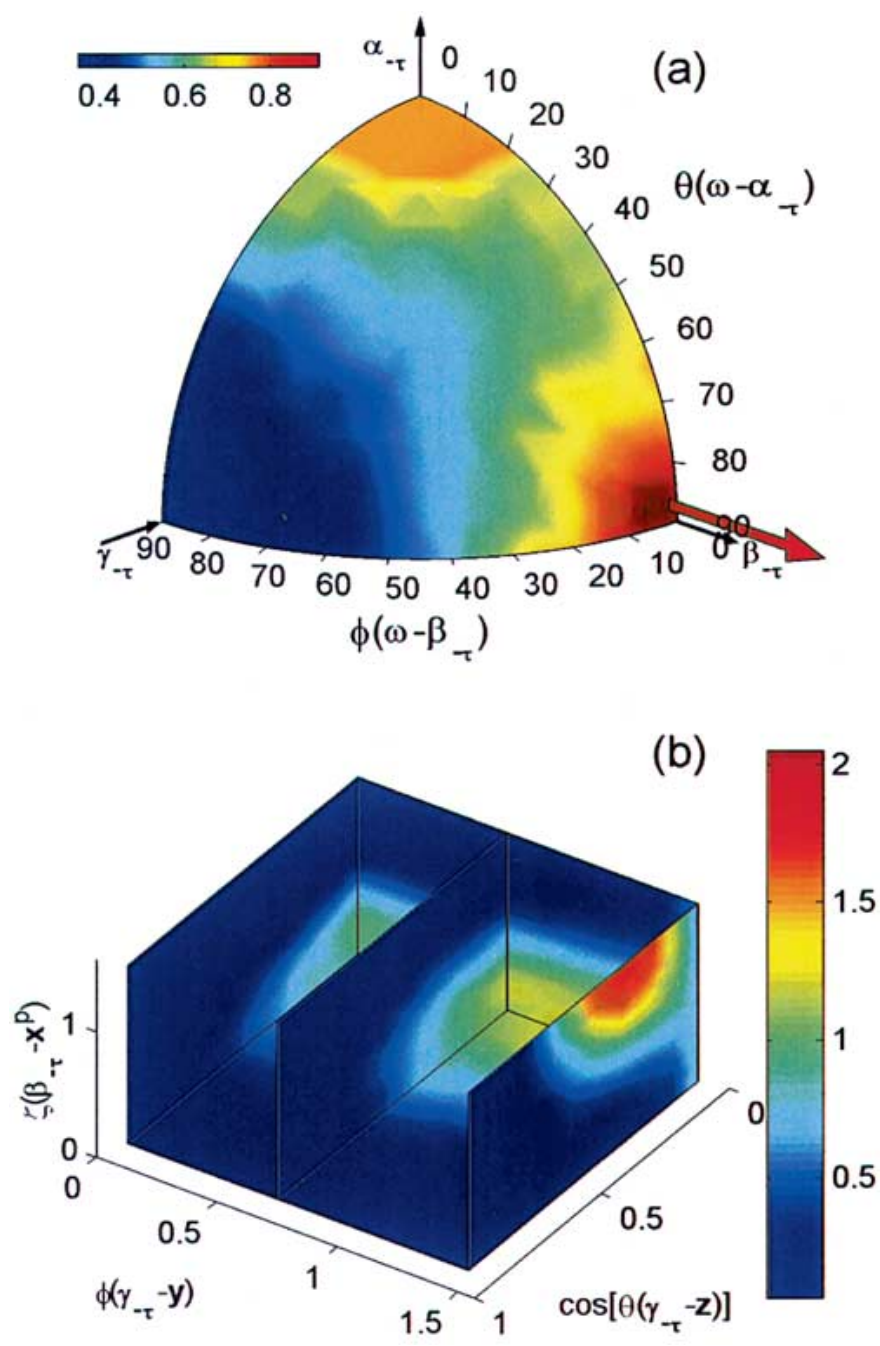

Figure 9. (a) Joint probability density function of two angles describing the orientation of filtered fluctuating vorticity vector in local negative SGS stress-tensor eigensystem. Filter scale is $\Delta=2 \mathrm{~m}$. The filtered fluctuating vorticity has a bimodal distribution giving two likely alignment configurations. The primary alignment is between filtered fluctuating vorticity and the intermediate eigendirection of the SGS stress, $\boldsymbol{\beta}_{-\boldsymbol{\tau}}$. The secondary alignment is vorticity and the extensive eigendirection of the negative SGS stress, $\boldsymbol{\alpha}_{-\boldsymbol{\tau}}$. (b) Joint PDF of three angles describing the orientation of SGS stress tensor eigensystem in mean flow frame of reference. Filter scale is $\Delta=2 \mathrm{~m}$. The mean flow streamwise direction is $x, z$ is the vertical axis, and $y$ is the transverse horizontal direction. The planes represent slices through the three-dimensional function. 
rotate $90^{\circ}$ about $\boldsymbol{\gamma}_{-\boldsymbol{\tau}}$. Neither configuration is consistent with the assumption of perfect alignment ((c) in Figure 10) used in the Smagorinsky model. This result is similar to the observed trends in HPIV and DNS data from Tao et al. (2002). It is noted that the preferred angles measured in the HPIV data are slightly higher than those obtained here: 32 as opposed to 28 degrees. Those in DNS are even higher, closer to 40 (S. Chen, personal communication, 2002 and Figure 14 of Tao et al., 2002). It is not clear what specific physical effects account for these quantitative differences in angles, but it is remarkable that the same basic alignment trends are observed in different turbulent flows of such disparate length scales, Reynolds numbers, and flow conditions.

To examine the effects of Taylor's hypothesis on the results, we divide the data set into two distinct regimes: High turbulence levels $\left|u^{\prime}\right| \geq 2 u_{*}$, and low turbulence levels $\left|u^{\prime}\right| \leq \frac{1}{2} u_{*}$. The alignment analysis is performed on these subsets of data, and we see that the resulting PDFs are quite similar (Figure 11a and b). The RMS difference between the joint PDF of the conditional results and the joint PDF from the entire data set is less than 0.035 in both cases, and gives confidence that the results obtained here are robust to the use of Taylor's hypothesis.

As discussed in Section 2, the focus of this study has been on the fluctuating quantities. But, for completeness, we include an analysis of stress-strain alignment with the filtered strain-rate elements that include their mean value. In this analysis, the only filtered strain-rate element with a nonzero mean is $\left\langle\tilde{S}_{13}\right\rangle \approx 0.15 \mathrm{~s}^{-1}$ (taken from Table I). This stress-strain alignment is shown in Figure 11c. As can be seen, the inclusion of the mean shear in the alignment analysis affects the strength of the peaks, but not their overall position. By comparison to the PDF of the fluctuating analysis (Figure 10), we see that the peak in probability corresponding to (a) is now stronger, and the peak in probability corresponding to (b) remains in the same position and is slightly weaker. Thus the only significant effect of the mean shear, associated with boundary-layer behaviour as discussed in Section 5.1, is to increase the probability of the primary peak.

\subsection{AlignMENT TRENDS OF THE MIXED MODEL}

As discussed in Section 2, the mixed model is sometimes used in LES. In terms of alignments, however, it turns out that we now must specify the ratio of two coefficients in order to study the alignments of the mixed model eigendirections relative to the measured SGS stress eigendirections. Alternatively, as was done in Tao et al. (2002), we can subtract from the real SGS stress tensor the nonlinear model multiplied by some coefficient. For instance, in Tao et al. (2002), it was suggested to subtract a Taylor expansion of the Galilean invariant Leonard stress $\Im_{i j}=\overline{\tilde{u}_{i} \tilde{u}_{j}}-\overline{\tilde{u}}_{i} \overline{\tilde{u}}_{j}$; where the overbar represents a second filtering at the same scale $\Delta$. Similar to the non-linear model, the Taylor series approximation to $\mathfrak{I}_{i j}$ yields

$$
\mathfrak{I}_{i j}^{*}=\frac{1}{12} \Delta^{2}\left(\frac{\partial \tilde{u}_{i}}{\partial x_{k}} \frac{\partial \tilde{u}_{j}}{\partial x_{k}}-\frac{1}{3} \frac{\partial \tilde{u}_{m}}{\partial x_{k}} \frac{\partial \tilde{u}_{m}}{\partial x_{k}} \delta_{i j}\right)
$$




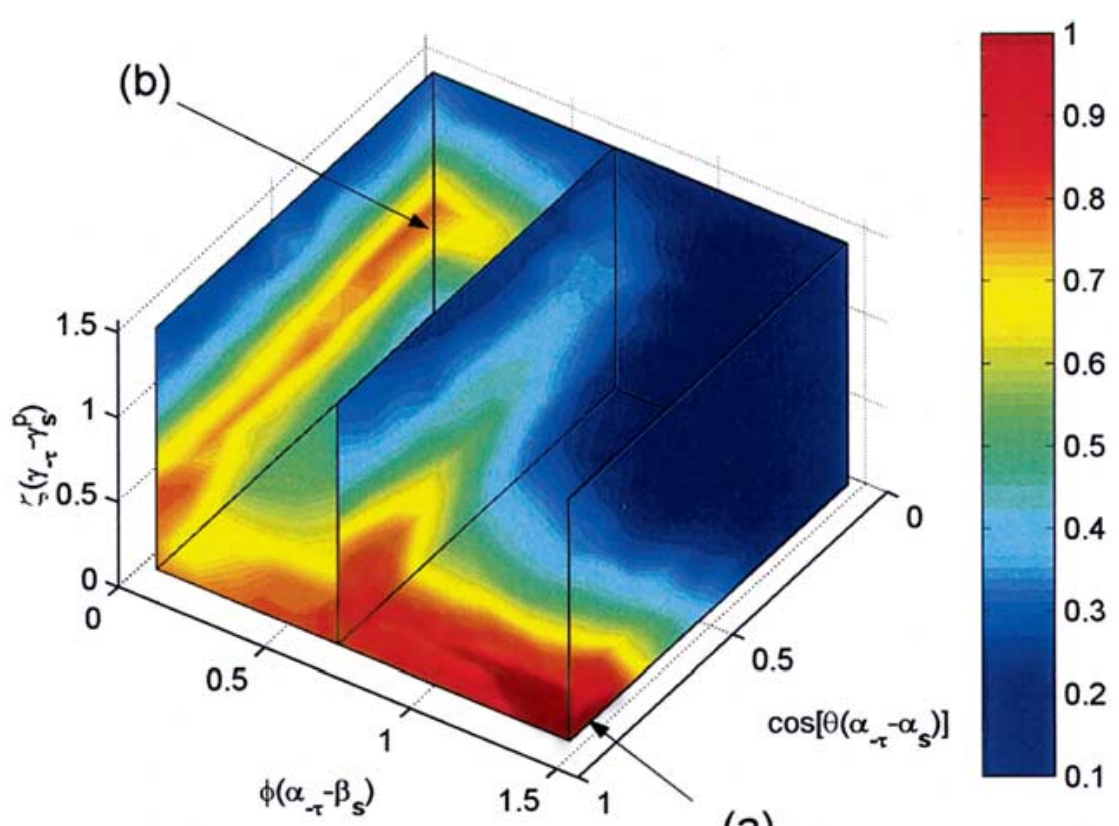

(a)

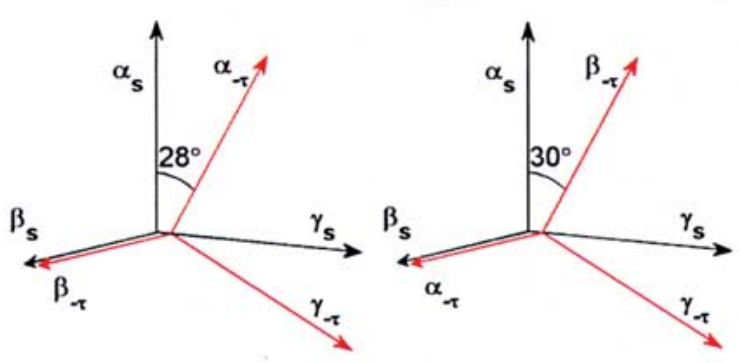

(a)

(b)

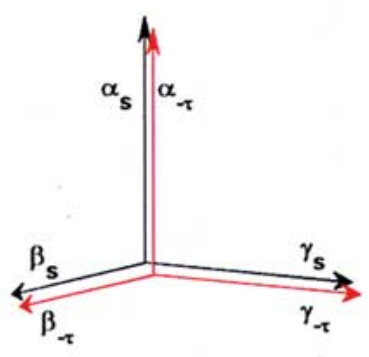

(c)

Figure 10. Joint probability density function of three angles describing the orientation of negative SGS stress tensor in filtered fluctuating strain-rate tensor eigensystem. Planes represent slices through the three dimensional function. A random alignment would correspond to a uniform 3-D distribution of joint probability equal to $(\pi / 2)^{-2} \approx 0.40$. Filter scale is $\Delta=2 \mathrm{~m}$. Interpretive sketches of each peak are given in (a) and (b). The eddy-viscosity alignment is given by interpretive sketch (c), corresponds to the line $\cos \left[\theta\left(\alpha_{-\tau}-\alpha_{\tilde{S}}\right)\right]=1$ and $\zeta\left(\gamma_{-\tau}-\gamma_{\tilde{S}}^{p}\right)=0^{\circ}$.

with a coefficient of 1/12. This term is often argued to be a good model for that part of the SGS stress that arises purely from the resolved velocity field in conjunction with smooth filters (such as the box and Gaussian filters used in the present analysis). If so, we can repeat the analysis of Section 5.2, subtracting Equation (11) from the SGS stress. The trace-free 'remainder stress', i.e.,

$$
\psi_{i j} \equiv\left(\tau_{i j}-\frac{1}{3} \tau_{k k} \delta_{i j}\right)-C_{n l} \Delta^{2}\left(\frac{\partial \tilde{u}_{i}}{\partial x_{k}} \frac{\partial \tilde{u}_{j}}{\partial x_{k}}-\frac{1}{3} \frac{\partial \tilde{u}_{m}}{\partial x_{k}} \frac{\partial \tilde{u}_{m}}{\partial x_{k}} \delta_{i j}\right)
$$


(a) $\left|u^{\prime}\right| \geq 2 u_{\star}$

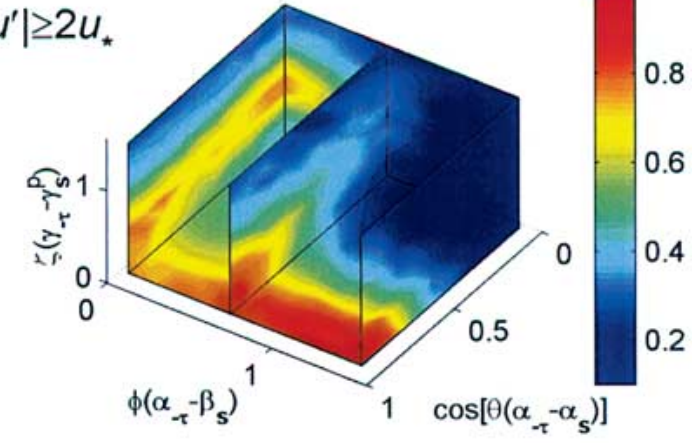

(b) $\left|u^{\prime}\right| \leq 0.5 u_{\star}$

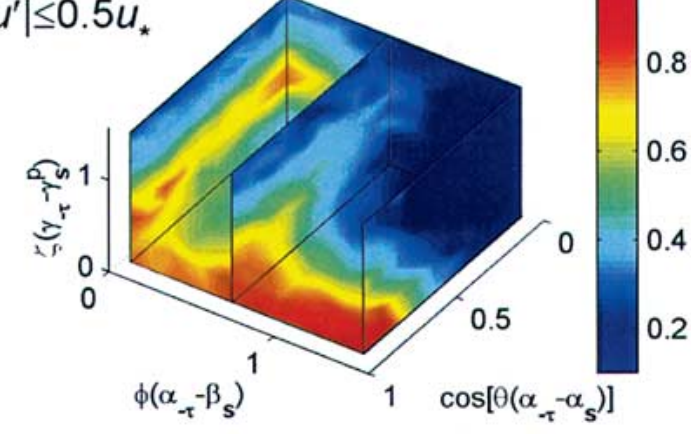

(c)

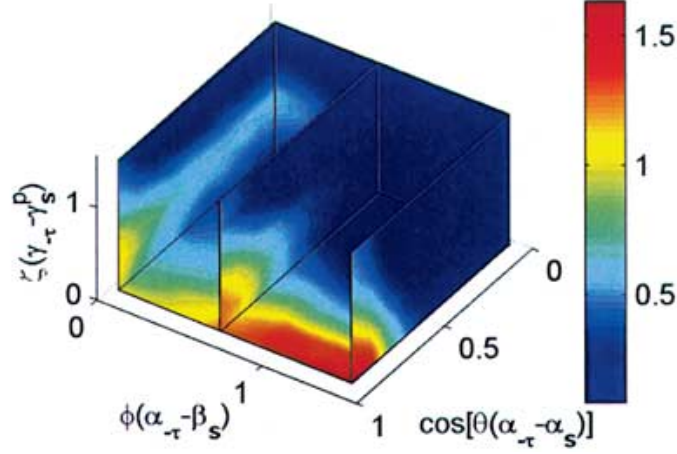

Figure 11. Joint probability density function of three angles describing the orientation of negative SGS stress tensor in filtered fluctuating strain-rate tensor eigensystem conditionally sampled on the high magnitudes $\left|u^{\prime}\right| \geq 2 u_{*}$ (a) and low magnitudes $\left|u^{\prime}\right| \leq \frac{1}{2} u_{*}$ (b) of the velocity fluctuations. The RMS difference between (a) and the PDF representing the entire data set is 0.0256 , and the RMS difference between (b) and the PDF representing the entire data set is 0.0347 . The deviation between the conditionally sampled PDFs and the PDF representing the entire data set is therefore about 1/30 of the total range of the joint PDF. (c) Joint probability density function of three angles describing the orientation of negative SGS stress tensor in the filtered fluctuating strain-rate tensor eigensystem, when the strain includes the mean (the only non-zero mean shear is $\left\langle\tilde{S}_{13}\right\rangle$ ). 
should now be well aligned with the strain-rate tensor basis if the mixed model provides realistic predictions of alignment trends. The choice of $C_{n l}$ is an issue since now the alignment results depend upon this value. In Tao et al. (2002), a value of $C_{n l} \approx 0.125$ (as opposed to $1 / 12$ of Equation (11)) was justified in terms of finite-difference evaluations of the gradients involved in the non-linear model. This was the natural approach since the data of Tao et al. (2002) were sufficient to permit 3-D spatial filtering.

To provide a more fruitful comparison with a SGS stress computed with a twodimensional (2-D) filter (as used in the present data analysis), we include only those terms in the calculation of $\psi_{i j}$ that are relevant to the filter operation. More specifically, if a 2-D filter $G(x, y)$ is used, then $\Im_{i j}=\overline{\tilde{u}_{i} \tilde{u}_{j}}-\overline{\tilde{u}}_{i} \overline{\tilde{u}}_{j}$; can be rewritten in terms of filtering convolutions as

$$
\begin{aligned}
\mathfrak{I}_{i j}= & \int_{-} \int_{-} G(\xi, \chi) \tilde{u}_{i}(x-\xi, y-\chi, z, t) \tilde{u}_{j}(x-\xi, y-\chi, z, t) \mathrm{d} \xi \mathrm{d} \chi \\
& -\int_{-} G(\xi, \chi) \tilde{u}_{i}(x-\xi, y-\chi, z, t) \mathrm{d} \xi \mathrm{d} \chi \\
& \times \int_{-} G(\xi, \chi) \tilde{u}_{j}(x-\xi, y-\chi, z, t) \mathrm{d} \xi \mathrm{d} \chi .
\end{aligned}
$$

The dimensionality of the filter operation dictates the variables over which $\tilde{u}_{i}$ is expanded. In the case of our 2-D filter operation, the Taylor series expansion of $\tilde{u}_{i}$ is

$$
\tilde{u}_{i}(x-\xi, y-\chi, z, t) \simeq \tilde{u}_{i}(x, y, z, t)-\xi \frac{\partial \tilde{u}_{i}}{\partial x}-\chi \frac{\partial \tilde{u}_{i}}{\partial y} .
$$

Using this expansion for $\tilde{u}_{i}$ in Equation (13), we obtain an equation for $\mathfrak{I}_{i j}^{*}$ that is relevant to the 2-D filtering employed in the present data analysis

$$
\mathfrak{I}_{i j}^{*}=\frac{1}{12} \Delta^{2}\left(\frac{\partial \tilde{u}_{i}}{\partial x} \frac{\partial \tilde{u}_{j}}{\partial x}+\frac{\partial \tilde{u}_{i}}{\partial y} \frac{\partial \tilde{u}_{j}}{\partial y}\right) .
$$

Equation (15) is similar to Equation (11), but lacks the inclusion of vertical gradients. Since the form of the convolution kernel dictates the Taylor series expansion of $\tilde{u}_{i}$, the final form (but not the constant $\Delta^{2} / 12$ ) of the Leonard stress approximation is also determined by the dimensionality of the filter operation. Using Equation (15), we redefine the trace free 'remainder stress' of Equation (12) as

$$
\begin{aligned}
\psi_{i j} \equiv & \left(\tau_{i j}-\frac{1}{3} \tau_{k k} \delta_{i j}\right)-C_{n l} \Delta^{2} \\
& \times\left(\frac{\partial \tilde{u}_{i}}{\partial x} \frac{\partial \tilde{u}_{j}}{\partial x}+\frac{\partial \tilde{u}_{i}}{\partial y} \frac{\partial \tilde{u}_{j}}{\partial y}-\frac{1}{3} \frac{\partial \tilde{u}_{m}}{\partial x} \frac{\partial \tilde{u}_{m}}{\partial x} \delta_{i j}-\frac{1}{3} \frac{\partial \tilde{u}_{m}}{\partial y} \frac{\partial \tilde{u}_{m}}{\partial y} \delta_{i j}\right) .
\end{aligned}
$$




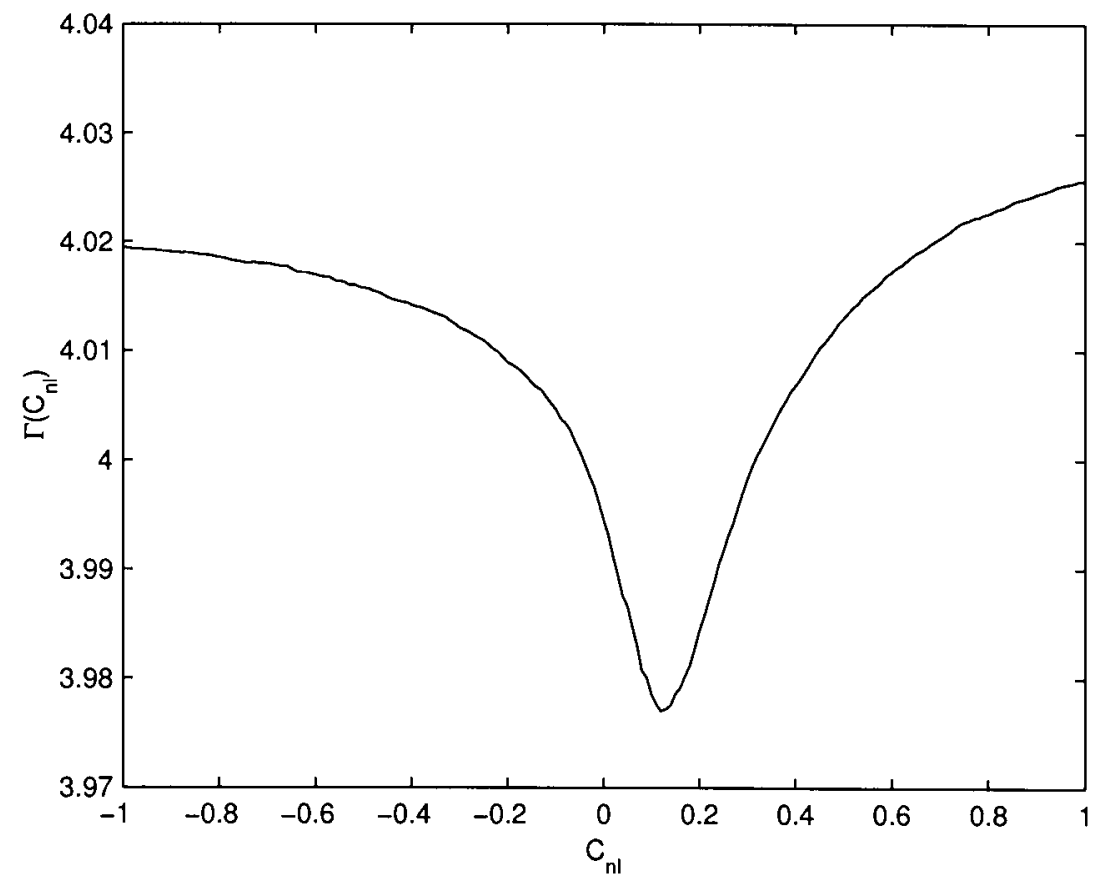

Figure 12. RMS difference from the ideal eddy viscosity alignment PDF and the measured PDF as a function of $C_{n l}$. The best value of $C_{n l}$ is 0.12 , for which the RMS difference is minimized.

To find an optimal value of $C_{n l}$ for the present data, we search for a value of $C_{n l}$ that minimizes the RMS difference between two joint PDFs of alignment angles. The first is the alignment between the remainder stress of Equation (16) and the filtered strain-rate tensor $P_{C_{n l}}(\cos \theta, \phi, \zeta)$. The second is the joint PDF of the ideal eddy-viscosity alignment $P_{\text {eddy }}=\delta(\cos \theta-1) \delta(\zeta-0)$. In this eddy-viscosity alignment, $\cos \left(\theta\left[\boldsymbol{\alpha}_{\tilde{\boldsymbol{S}}}-\boldsymbol{\alpha}_{-\tau}\right]\right)=1$ at all times (i.e., $\boldsymbol{\alpha}_{\tilde{S}}$ and $\boldsymbol{\alpha}_{-\tau}$ are always aligned) and $\left.\zeta\left[\boldsymbol{\gamma}_{\tilde{S}}^{p}-\boldsymbol{\gamma}_{-\tau}\right]\right)=0^{\circ}$ at all times (i.e., given the alignment of $\boldsymbol{\alpha}_{\tilde{S}}$ and $\boldsymbol{\alpha}_{-\tau}, \boldsymbol{\gamma}_{\tilde{S}}$ and $\gamma_{-\tau}$ are always aligned). In the data analysis, $P_{\text {eddy }}=(2 / 5) \pi^{2}$ in the 10 bins closest to $\cos \theta=1$ and $\zeta=0$. The RMS difference between both PDFs is defined as

$\Gamma\left(C_{n l}\right)=\left\{\int_{0}^{\pi / 2} \int_{0}^{\pi / 2} \int_{0}^{1}\left(P_{C_{n l}}(\cos \theta, \phi, \zeta)-P_{\text {eddy }}\right)^{2} \mathrm{~d}(\cos \theta) \mathrm{d} \zeta \mathrm{d} \phi\right\}^{1 / 2}$.

Figure 12 presents a plot of $\Gamma\left(C_{n l}\right)$ that can be interpreted as a measure of the disparity between the alignment PDF resulting from a particular value of $C_{n l}$ in the remainder stress, and the ideal eddy-viscosity alignment $P_{\text {eddy }}$. Although the actual values of $\Gamma\left(C_{n l}\right)$ vary over a small range, the resulting PDFs for different values of $C_{n l}$ are visibly different. From Figure 12, it is clear that a value of $C_{n l}=0.12$, similar to the value reported by Tao et al. (2002), gives an alignment of the remainder stress, $\psi_{i j}$ with the strain-rate eigenvectors that is closest to eddy viscosity. 


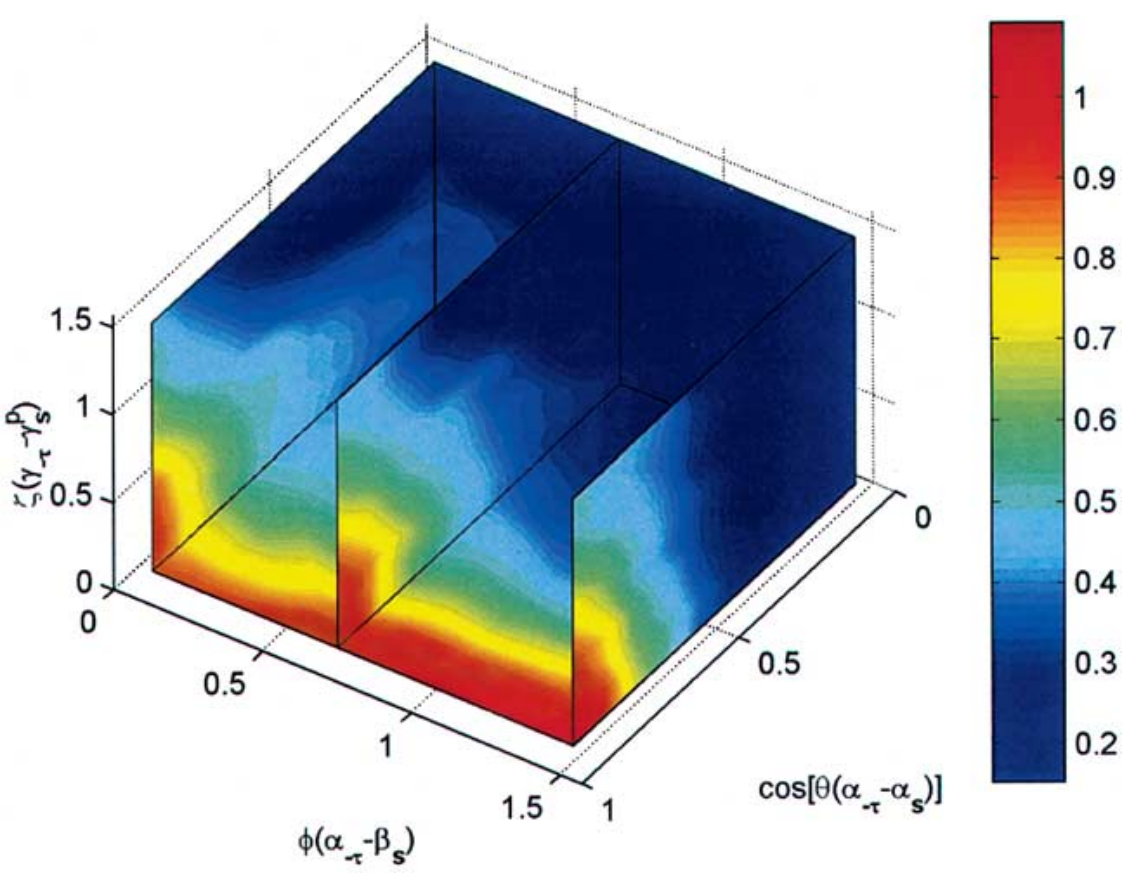

Figure 13. Joint probability density function of three angles describing the orientation of negative SGS stress tensor with nonlinear model part subtracted (as in Equation (8)) with $C_{n l}=0.12$. The orientation is with respect to the filtered fluctuating strain-rate tensor eigensystem. A random alignment would correspond to a uniform 3-D distribution of joint probability equal to $(\pi / 2)^{-2} \approx 0.40$. The collapse towards alignment of $\theta\left(\boldsymbol{\alpha}_{\tilde{\boldsymbol{S}}}-\boldsymbol{\alpha}_{-\boldsymbol{\tau}}\right) \approx 0$ shows that alignment trends of the remainder of the SGS stress are well reproduced by an eddy-viscosity closure, and that a mixed model is well supported by present results.

The alignment trend observed between the remainder stress with the optimal value of $C_{n l}=0.12$ and filtered strain rate is shown in Figure 13. The bimodal secondary peak that was visible in Figure 10 has disappeared, and the primary peak has moved so that the most likely value of $\cos \left(\theta\left[\boldsymbol{\alpha}_{\tilde{\boldsymbol{S}}}-\boldsymbol{\alpha}_{-\tau}\right]\right)=1$ and $\zeta\left[\boldsymbol{\gamma}_{\tilde{\boldsymbol{S}}}^{p}-\right.$ $\left.\left.\gamma_{-\tau}\right]\right)=1$ this now corresponds to the eddy-viscosity alignment for the remainder stress.

\section{Summary and Discussion}

We have analyzed field experimental data obtained in the atmospheric surface layer using tools from statistical geometry. Specifically, the field data consisted of velocity measurements from sonic anemometer arrays that permit spatial filtering. Such filtering separates large from small scales so that the data can be interrogated in the context of large eddy simulation (LES). Time series of various quantities relevant to LES were evaluated from the data, such as SGS stress tensor, filtered vorticity 
vector and filtered strain-rate tensor. The PDFs of strain-state parameters indicated that the preferred fluid deformation is axisymmetric extension and the preferred SGS stress state is axisymmetric contraction. The filtered fluctuating vorticity has preferred alignments with the mean vorticity, with the streamwise direction (e.g., streamwise vortices), and with the intermediate strain-rate eigenvector.

The alignment between eigenvectors of the SGS stress and filtered strain rate was used to test the eddy-viscosity model formulation, and a bimodal distribution was observed. Moreover, we observed the collapse of the alignment to the eddyviscosity model when a portion of the Leonard stress is taken from the SGS stress, in support of the mixed model. Good qualitative agreement with previous results in laboratory experimental studies and numerical results at much lower Reynolds numbers suggest that the present observations are indicative of universal features of turbulent flows. However, quantitative differences (such as in exact values of alignment angles) suggest that more research is needed to better understand underlying physics and to provide quantitative parameterizations. It should also be stressed that in this paper the focus was on purely geometric alignment trends. Improved SGS parameterizations will also require a better understanding of how to model the magnitudes of the tensor eigenvalues.

\section{Acknowledgements}

The authors wish to thank their colleagues Markus Pahlow and Jan Kleissl (Johns Hopkins University), Fernando Porté-Agel (University of Minnesota), William Eichinger (University of Iowa) for fruitful discussions and invaluable assistance in the field. We also thank Gabriel Katul (Duke University) and Jay Ham (Kansas State University) for the loan of sonic anemometers. This work has been supported by NSF-ATM 0130766, NSF-EAR and an EPA STAR fellowship.

\section{References}

Ashurst, W. T., Kerstein, A. R., Kerr, R. M., and Gibson, C. H.: 1987, 'Alignment of Vorticity and Scalar Gradient with Strain Rate in Simulated Navier-Stokes Turbulence', Phys. Fluids 30, 2343.

Bell, J. H. and Metha, R. D.: 1992, 'Measurements of the Streamwise Vortical Structures in a Plane Mixing Layer', J. Fluid Mech. 239, 213-248.

Bernal, L. P. and Roshko, A.: 1986, 'Streamwise Vortex Structure in Plane Mixing Layers', J. Fluid Mech. 170, 499-525.

Cantwell, B. J.: 1981, 'Organized Motion in Turbulent Flow', Annu. Rev. Fluid Mech. 13, 457-513.

Cerutti, S. and Meneveau, C.: 2000, 'Statistics of Filtered Velocity in Grid and Wake Turbulence', Phys. Fluids 12, 1142-1165.

Clark, R. G., Ferziger, J. H., and Reynolds, W. C.: 1979, 'Evaluation of Subgrid Models Using an Accurately Simulated Turbulent Flow', J. Fluid Mech. 91, 1-16.

Finnigan, J.: 2000, 'Turbulence in Plant Canopies', Annu. Rev. Fluid Mech. 32, 519-571.

Germano, M.: 1986, 'A Proposal for a Redefinition of the Turbulent Stresses in the Filtered NavierStokes Equations', Phys. Fluids 29, 2323. 
Lasheras, J. C. and Choi, H.: 1988, 'Three-Dimensional Instability of a Plane Free Shear Layer: An Experimental Study of the Formation and Evolution of Streamwise Vorticies', J. Fluid Mech. 189, 53-86.

Leonard, A.: 1974, 'Energy Cascade in Large-Eddy Simulations of Turbulent Fluid Flows', Adv. Geophys. 18, 237.

Lilly, D. K.: 1967, 'The Representation of Small-Scale Turbulence in Numerical Simulation Experiments', Proceedings of the IBM Scientific Computing Symposium on Environmental Sciences, pp. 195-210.

Lin, C. L.: 1999, 'Near-Grid-Scale Energy Transfer and Coherent Structures in the Convective Planetary Boundary Layer', Phys. Fluids 11, 3482-3494.

Liu, S., Katz, J., and Meneveau, C.: 1994, 'On the Properties of Similarity Subgrid-Scale Models as Deduced from Measurements in a Turbulent Jet', J. Fluid Mech. 275, 83.

Lund, T. S. and Rogers, M. M.: 1994, 'An Improved Measure of Strain State Probability in Turbulent Flows', Phys. Fluids 6, 1838-1847.

Meneveau, C. and Katz, J.: 2000, 'Scale Invariance and Turbulence Models for Large-EddySimulation', Annu. Rev. Fluid Mech. 319, 353-385.

Pahlow. M., Parlange, M. B., and Porté-Agel. F.: 2001, 'On Monin-Obukhov Similarity in the Stable Atmospheric Boundary Layer', Boundary-Layer Meterol. 99, 225-248.

Piomelli, U.: 1999, 'Large Eddy Simulation: Achievements and Challenges', Prog. Aerosp. Sci. 35, 355-362.

Porté-Agel, F., Meneveau, C., and Parlange, M. B.: 1998, 'Some Basic Properties of the Surrogate Subgrid-Scale Heat Flux in the Atmospheric Boundary Layer', Boundary-Layer Meteorol. 88, 425-444.

Porté-Agel, F., Meneveau, C., and Parlange, M. B.: 2000a, 'A Scale Dependent Dynamic Model for Large Eddy Simulation: Application to a Neutral Atmospheric Boundary Layer', J. Fluid Mech. 415, 261-284.

Porté-Agel, F., Parlange, M. B., Meneveau, C., Eichinger, W. E., and Pahlow, M.: 2000b, 'SubgridScale Dissipation in the Atmospheric Surface Layer: Effects of Stability and Filter Dimension', J. Hydrometeorol. 1, 75-87.

Porté-Agel, F., Parlange, M. B., Meneveau, C., and Eichinger, W. E.: 2001a, 'A Priori Field Study of the Subgrid-Scale Heat Fluxes and Dissipation in the Atmospheric Surface Layer', J. Atmos. Sci. 15, 2673-2697.

Porté-Agel, F., Pahlow, M., Meneveau, C., and Parlange, M. B.: 2001b, 'Atmospheric Stability Effect on Subgrid Scale Physics for Large-Eddy Simulation', Adv. Water Resour. 24, 1085-1102.

Stevens, B. and Lenschow, D. H.: 2001, 'Observations, Experiments, and Large Eddy Simulation', Bull. Amer. Meteorol. Soc. 82, 283-294.

Tao, B., Katz, J., and Meneveau, C.: 2002, 'Statistical Geometry of Subgrid-Scale Stresses Determined from Holographic Particle Image Velocimetry Measurements', J. Fluid Mech. 457, $35-78$.

Tong, C., Wyngaard, J. C., and Brasseur, J. G.: 1999, 'Experimental Study of the Subgrid-Scale Stresses in the Atmospheric Surface Layer', J. Atmos. Sci. 56, 2277-2292.

Tsinober, A., Kit, E., and Dracos, T.: 1992, 'Experimental Investigation of the Field of VelocityGradients in Turbulent Flows', J. Fluid Mech. 242, 169-192. 
